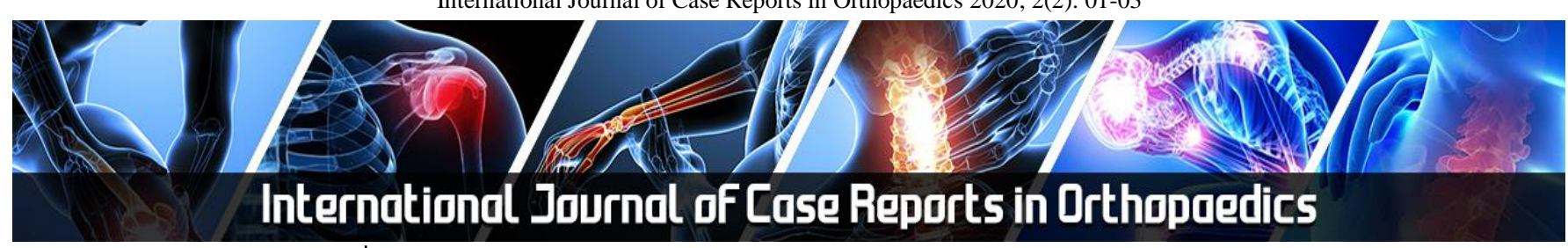

E-ISSN: 2707-8353 P-ISSN: 2707-8345 IJCRO 2020; 2(2): 01-03 Received: 03-05-2020 Accepted: 08-06-2020

Dr. Ibrahim M Hussein MSc, FEBOT, MRCPS Orthopaedic Surgeon, Orthopaedic Department, King Faisal Medical Complex, Taif, Saudi Arabia
Corresponding Author: Dr. Ibrahim M Hussein MSc, FEBOT, MRCPS Orthopaedic Surgeon, Orthopaedic Department, King Faisal Medical Complex, Taif, Saudi Arabia
Case Report

\section{Floating hip: A unique pattern of injury}

\section{Dr. Ibrahim M Hussein}

DOI: $\underline{\text { https://doi.org/10.22271/27078345.2020.v2.i2a.18 }}$

\begin{abstract}
Floating hip is a rare injury, usually involved in high energy trauma. The classification and the guidelines for management of such rare injury is debatable in the literatures. I'm reporting a case of a 37-year-old male presented to my hospital with a transverse and posterior wall fracture of the right acetabular, ipsilateral proximal femoral shaft fracture and posterior hip dislocation. To my knowledge, this case is unique in presentation.

Closed reduction failed, hence an open reduction internal fixation of his hip fracture-dislocation and acetabular fracture by plate and screws were done.
\end{abstract}

Keywords: Floating hip, Acetabulum Fracture, Femur Fracture

\section{Introduction}

A floating joint term defined as discontinuing the bone above and the bone below the joint. 'floating hip' is defined as a fracture of the pelvis or acetabulum with a concomitant femur fracture ${ }^{[1,2]}$. This combination of injuries is uncommon and estimated to occur once in every 10000 fractures ${ }^{[3,4]}$. There is no consensus classification for such rare injury. Existing studies have classified the injury into two major types according to the forms of injury to the pelvis or acetabulum, and subsequently, another type with injuries to both the pelvic ring and acetabulum was added to the classification. Meanwhile, Nathan classified type A as femur fracture occurring together with pelvic ring fracture and type B as occurring together with acetabulum fracture, and claimed that type B was a true form of floating hip ${ }^{[4]}$. Müller et al. added type $\mathrm{C}$ classification as femoral, pelvic ring, and acetabular fractures occurring simultaneously [3]. However, this patient sustained a fracture of proximal femoral shaft accompanied by transverse with posterior wall acetabular fractures and extruded head of femur outside the acetabulum in the gluteal area. According to existing classification methods, this patient has unique fracture pattern that is needed to be included in appropriate classification system, I'm reporting my treatment experience in this case and proposing that unique dislocation pattern to be included in the classification of the floating hip injury types based on this case report.

\section{Case Report}

A 37 years male patient, presented to our Emergency Department in king Faisal Medical Complex Hospital in Taif, Saudi Arabia, after involving in road traffic accident (RTA) and sustaining fracture dislocation of the right hip of very rare presentation. Patient was conscious and oriented with normal vital signs and Glasgow Coma Scale (GCS) was 15. Advanced Trauma Life Support (ATLS) protocol was followed. On orthopaedic examination, there was a deformity of the whole right lower limb without neurovascular complications. After full imaging, patient was diagnosed with intertrochanteric fracture, posterior dislocation of the right hip in addition to transverse acetabular fracture with posterior wall. A trial of closed reduction was unsuccessful because of extruded femoral head and neck outside the acetabulum in the right gluteal area. After a discussion with the patient, regarding the rare diagnosis and bad prognosis including the risk of non-viability of femoral head, an informed consent for surgery was signed and prophylactic antibiotic Cefazolin was administered. In the Operative Theater, the patient was positioned in the lateral decubitus, my surgical plan was to use one incision for approaching all fractures through one extended posterior approach to the right hip and acetabulum, the proximal femoral piece found extruded under the gluteus maximus muscle which was reduced 
manually into the acetabulum after clearing it from intraarticular bone fragments in order to clear the field to see the fracture acetabulum. I started with fixation of the acetabulum by double $3.5 \mathrm{~mm}$ reconstruction plate, one for posterior column and one for posterior wall. Then the proximal femoral piece was fixed into femoral shaft by inter-fragmentary 2 screws and Dynamic Hip Screw (DHS) where I prefer to make the Tip Apex Distance (TAD) longer than usual to avoid acetabular attrition by threads of lag screw after an inevitable AVN and collapse of femoral head later on post-operatively being completely detached from any soft tissue attachments and migrated under the gluteal maximus; in addition to the postoperative non-weight bearing policy for three months. Then, reconstruction of the avulsed and comminuted greater trochanter was done by adding Trochanter Stabilizing Plate (TSP) to DHS plate, in addition to application of a tension band wiring to the fractured greater trochanter, an augmentation of the tension band fixation by inter-fragmentary screws were added to hold the trochanter being a-four-piece of bone. The stability of fracture was checked; additionally, the quality of reduction was checked by $\mathrm{C}$-Arm image and was satisfactory. Washing, irrigation of wound and insertion of suction drain, followed by closure of wound in layers. Postoperative period passed without wound infection or $\mathrm{N}-\mathrm{V}$ complications. Pt was discharged from the hospital to his home after one week and an Out Patient Clinic (OPD) appointment after six weeks for follow-up was scheduled. The patient was instructed about the postoperative rehabilitation that including non-weight bearing for right leg for three months.

\section{Preoperative X Ray}
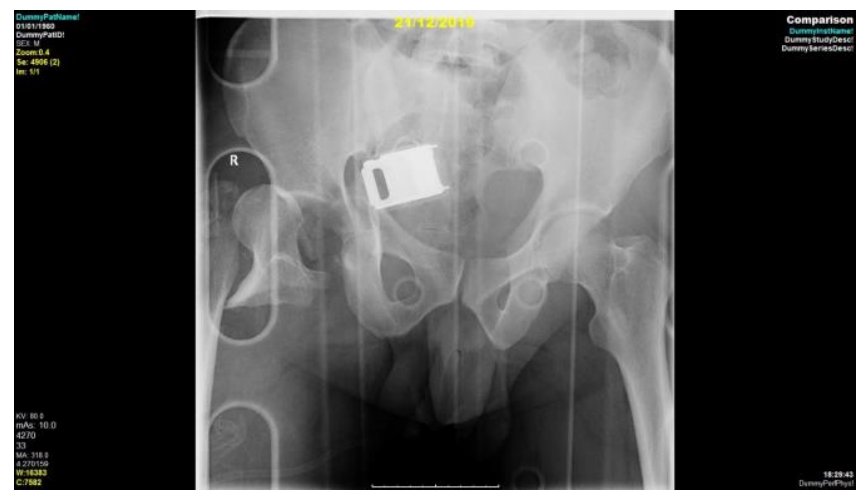

\section{Pre-Operative CT Scans}

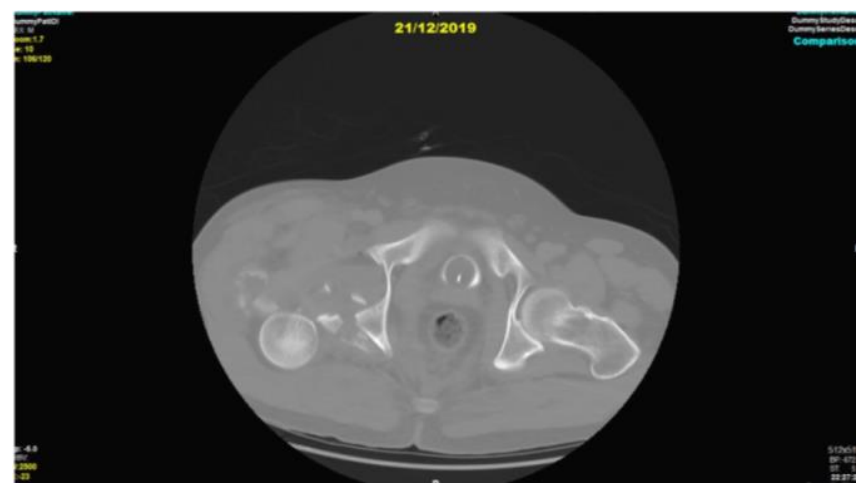

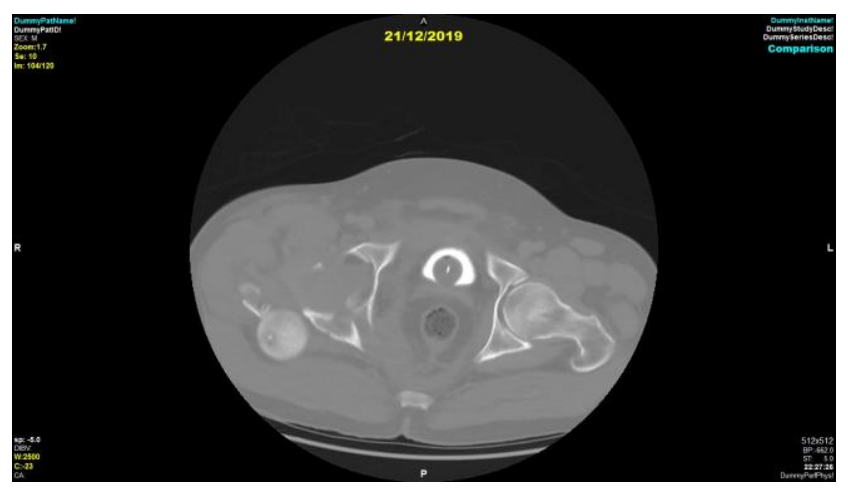
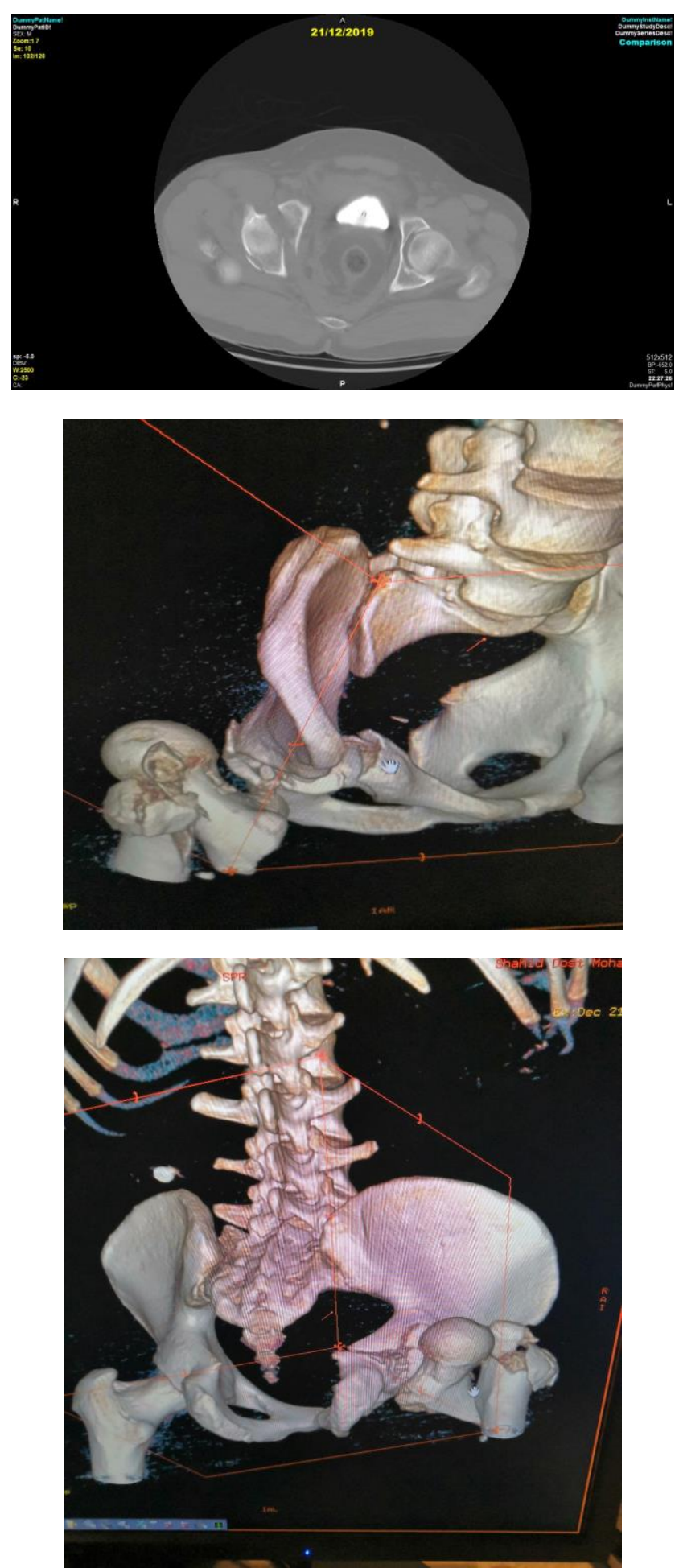


\section{Postoperative X Rays}
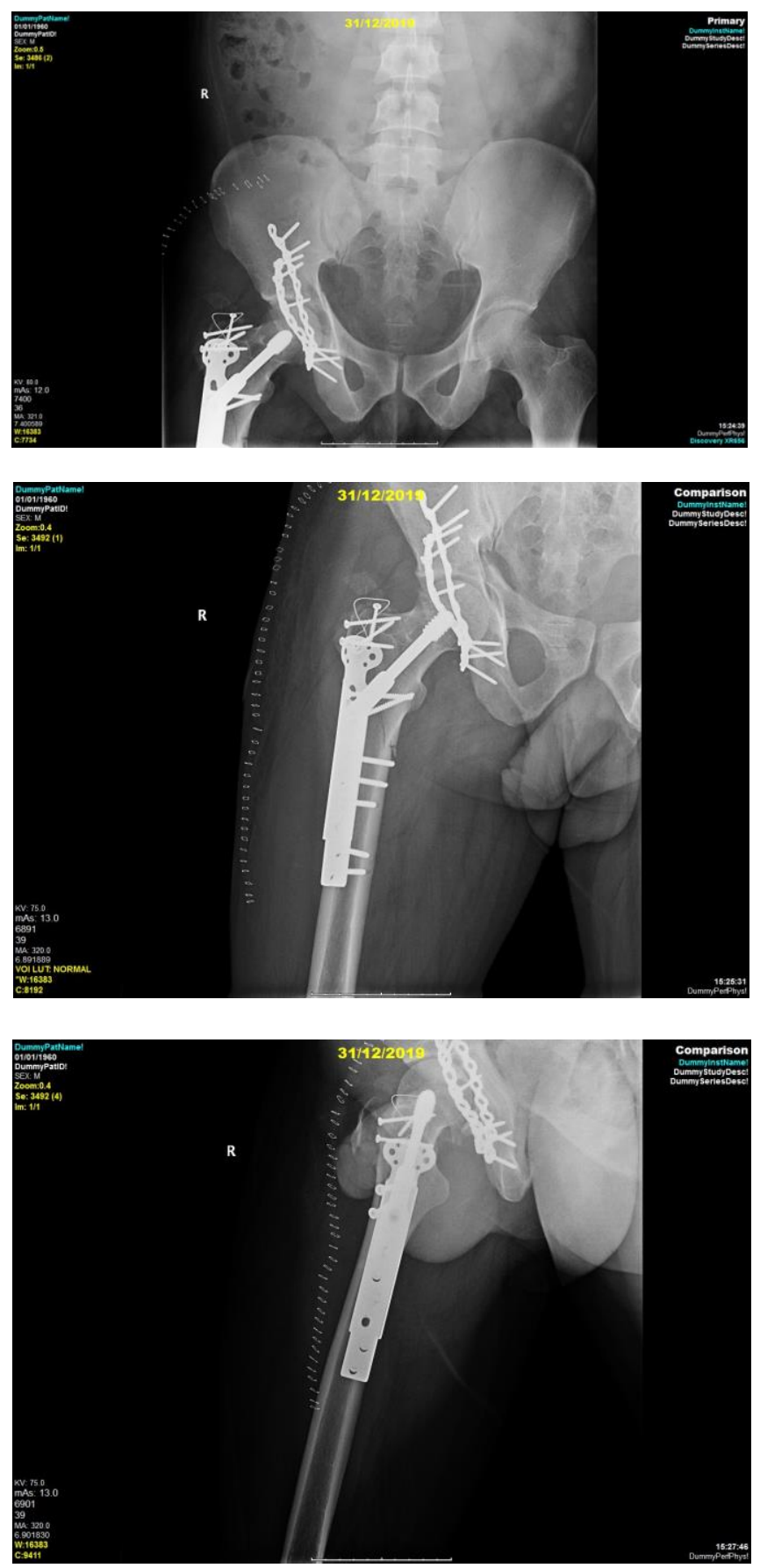

\section{Discussion}

The literatures discussing the floating hip injuries focus on femoral fixation preceding the definitive management of pelvic and acetabular fractures. However, many of these reports did not exhibit an associated hip dislocation. My patient presented to my hospital, after $10 \mathrm{~h}$ after accident, referred from a peripheral hospital, with the right hip dislocation which mandated emergent open reduction to reduce the risk of avascular necrosis to the femoral head. The appropriate surgical approach for such case was a dilemma regarding one to two incision and which fracture to be fixed first. The extended Kocher-Langenbeck approach was chosen to provide an excellent visualization of the posterior acetabular fracture, reduction of the femoral head into the acetabulum and fixation of femoral shaft fracture. Early internal fixation of fractures and reduction of dislocation permits mobilization and correlates with improved clinical outcomes. This unique injury, being an isolated injury, in a generally stable patient, permitted operative hip reduction within $13 \mathrm{~h}$ of injury with definitive fixation of all fractures.

Preoperatively, there were no neurological or vascular injuries in our case, the soft tissue damage was not significant as well. Liebergall indicated that early fixation on pelvic and femoral fractures is needed for floating hip fracture cases, during which time, fixing the femur first can be useful for intra-articular acetabular fracture reduction through traction ${ }^{[2]}$. However, in my patient the fractured, dislocated and extruded femoral head made closed reduction impossible. Tiedeken emphasized the need for emergency open reduction within $6 \mathrm{~h}$ in hip dislocation cases due to possible necrosis of the femoral head from avascular state [1]. However, my patient presented to the hospital after 10 hours; in addition, the proximal femoral fragment was completely detached from any soft tissue attachment and the hip capsule found shattered. Based on the above, I considered that AVN and femoral head collapse will be inevitable. Consequently, the idea was to make the lag screw little short (long TAD) because patient was young, his bones in good quality and he will not walk before three months. The idea behind that, was to delay the anticipated degenerative changes in the acetabulum after proposed inevitable head collapse with subsequent protrusion of the threads of lag screw. This idea will need further study to explain if there are any exceptions to commit with ideal TAD because most of literatures discuss TAD in usual intertrochantric fracture in old patients with osteoporotic bone which is not our case. Of note, in this rare, the patient did not have any sciatic nerve injury despite severity of injury with totally extruded femoral head and neck in gluteal region; as well as, the postoperative period passes without sciatic nerve palsy too which made this patient's presentation is unique in regard to severity of injury.

\section{Conflicts of Interest Statement}

The author declare that there is no conflict of interests regarding the publication of this paper

\section{Funding/Support Statement}

The author declare that there is no funding/support regarding the publication of this paper

\section{References}

1. Nathan C. Tiedeken, Vilas Saldanha, John Handal and James Raphael. The irreducible floating hip: a unique presentation of a rare injury, Journal of Surgical Case reports, 2013, 10

2. Liebergall M, Mosheiff R, Safran O, Peyser A, Segal D. The floating hip injury: patterns of injury. Injury. 2002; 33:717-22.

3. Muller EJ, Siebenrock K, Ekkernkamp A, Ganz R, Muhr G. Ipsilateral fractures of the pelvis and femurfloating hip? Arch of Orthop Trauma Surg 1999; 119:179-83.

4. Wiltberger BR, Mitchell CL, Hedrick DW. Fracture of the femoral shaft complicated by hip dislocation-a method of treatment. J Bone Joint Surg Am. 1948; 30A:225-8.

5. Chi-Chuan W, Chun-Hsuing S, Lih-Huei C. Femoral shaft fractures complicated by fracture-dislocations of the ipsilateral hip. J Trauma. 1993; 34:70-5.

6. Il Yong Han, Gyu Min Kong, Floating Hip a case Report, J Orthopaedics Trauma Surgery and Related research, 2017e-ISSN, 2449-9145. 